PROCEEDINGS OF THE

AMERICAN MATHEMATICAL SOCIETY

Volume 130, Number 12, Pages 3619-3629

S 0002-9939(02)06585-1

Article electronically published on May 1, 2002

\title{
BRAIDING MINIMAL SETS OF VECTOR FIELDS
}

\author{
MAXIME LAGRANGE
}

(Communicated by Michael Handel)

\begin{abstract}
We extend a classical but fundamental theorem of knot and braid theories to describe the geometry of nonsingular minimal sets of 3-dimensional flows.
\end{abstract}

\section{INTRODUCTION}

The idea that periodic orbits are crucial when trying to understand global dynamics in dimension three is certainly due to Henri Poincaré (see $\mathrm{Po}]$ ). Since then, this idea has been widely improved and has been very fruitful in the study of homoclinic orbits to a periodic one by Birkhoff and Smale (see [Bi], $[\mathrm{Sm}]$ ).

In the last decades, a more specific point of view has been standing out. It is based on the fact that a periodic orbit of a flow on the 3 -sphere $\mathbb{S}^{3}$ forms a knot, i.e. the embedding of an oriented circle into $\mathbb{S}^{3}$ (see $[\mathrm{Mu}$ for a survey of knot and braid theories). For instance, Birman and Williams (BiWi1] and [BiWi2 ] investigated knot and link types of periodic orbits in flows on a 3-manifold. Some recent works show that the isotopy classes of knots and links represented by a finite set of periodic orbits of a three-dimensional vector field provide interesting information about global dynamics. For instance, Wada (see Wa ) has shown that the type of knots that can be reached as periodic orbits of Morse-Smale flows on $\mathbb{S}^{3}$ are of a very particular kind, such as connected sums of iterated torus knots (see $[\mathrm{Mu}]$ ). More recently, Casasayas, Martinez Alfaro and Nunes (see [CMAN]) have related Bott integrable Hamiltonian flows to nonsingular Morse-Smale flows in order to show that the same result holds for periodic orbits of a Bott-integrable Hamiltonian flow on a nondegenerate constant-energy three-manifold.

In the particular case of suspensions of diffeomorphisms of the disk, the topological object that arises naturally is closed braids. It has been used for instance by Gambaudo, van Strien and Tresser (see [GST1]) to describe the set of periodic orbits of $C^{1}$-diffeomorphisms of the disk with zero entropy. Also using techniques coming from closed braids, some generalizations of Sarkovskiı's theorem to diffeomorphisms of the disk have been proved: in some cases the existence of a periodic orbit of period 3 implies the existence of periodic points with any period (see GST2 and $[\mathrm{Ko}]$ ).

Since closed braids are conjugacy classes of Artin braids (see [Mu again) and then possess an algebraic structure, it is easier to work with closed braids than with

Received by the editors July 13, 2001.

2000 Mathematics Subject Classification. Primary 37Cxx, 57Mxx.

Key words and phrases. Dynamical systems, knot theory, minimal sets.

(C)2002 American Mathematical Society 
knots. Actually, it is one of the reasons why the dynamics of diffeomorphisms of the disk is better understood than the one of three-dimensional flows (see [Bo]).

However, these two notions are closely related. Indeed, Alexander (see [Al]) showed that for every link $L$ in $\mathbb{S}^{3}$ there exists an ambient isotopy which sends $L$ into the standard revolution solid torus $\mathcal{T}$ so that its image is transverse to the fibers of $\mathcal{T}$, thus changing the link into a closed braid.

Unfortunately, periodic orbits do not always carry enough information and the following two results show the limit of this approach. On one hand, there exists a flow whose set of periodic orbits contains all knots and links (see [GhHo]). On the other hand, the Seifert question, that wonders whether a smooth flow on $\mathbb{S}^{3}$ possesses a periodic orbit, has been answered negatively (see [Ku or $\mathrm{Gh}$ for more details): there exist smooth aperiodic vector fields on the 3 -sphere.

So, it seems natural to try to generalize to a more general class of orbits the topological notions defined for periodic orbits via knot theory.

From a statistical point of view, a good candidate should be the supports of ergodic measures. This approach has been done by Arnold (see [ArKh]) in hydrodynamics to study the energy relaxation of a flow. He showed that the way orbits of a smooth divergence-free vector field are asymptotically linked in the 3sphere gives a reduction of its energy. If $E_{2}(-)$ denotes the quadratic energy, we get $E_{2}(X) \geq C\left|\mathcal{A}_{X}\right|$, where $\mathcal{A}_{X}$ is the asymptotic Hopf invariant, which is invariant under the volume preserving change of variables. Following this idea, Gambaudo and Ghys (see GaG1] and GaG2]) developed techniques to generalize knot invariants, such as linking a number or signature, to invariant measures of vector fields.

From a topological point of view, the appropriate set of orbits that naturally appears is a minimal set:

Definition 1.1. A nonempty compact set is minimal if it is a union of trajectories of the flow and if none of its proper subsets has this property.

The main advantage of considering minimal sets instead of periodic orbits is that every vector field on a compact manifold admits a minimal set (it is an easy consequence of Zorn's lemma; see [KaHa for details concerning minimal sets and dynamics).

Moreover a minimal set can be much more complicated than periodic orbits. For instance, a minimal set homeomorphic to the Denjoy set appears in the study of the Kuperberg attractor whose structure has not yet been completely understood (see $[\overline{\mathrm{Ku}}]$ ). In many cases (think for instance about the suspension of the Denjoy counterexample) the minimal set is a solenoid, i.e.:

Definition 1.2. A minimal set $M$ of a vector field $X$ is a solenoid if it is locally homeomorphic to the product of the Cantor by an interval.

The aim of this article is to ask whether there is an analogous tool to knots and braids which may be relevant for the study of minimal sets of smooth vector fields of euclidian 3-dimensional spaces.

More precisely, we will consider a minimal set $M$ of a vector field in $\mathbb{S}^{3}=$ $\left\{(x, y, z, t) \in \mathbb{R}^{4} \mid x^{2}+y^{2}+z^{2}+t^{2}=1\right\}$ or $\mathbb{R}^{3}$, different from a singularity. As in knot theory we will try to braid it.

Recall the parametrization defining the standard revolution torus $\mathcal{T}$ in $\mathbb{R}^{3}$ :

$$
\begin{aligned}
{[0,1] \times[0,2 \pi] \times[0,2 \pi] } & \rightarrow \mathbb{R}^{3} \\
(\rho, \phi, \psi) & \mapsto((2+\cos (\phi)) \cos (\psi),(2+\cos (\phi)) \sin (\psi), \sin (\phi)) .
\end{aligned}
$$




\section{Definition 1.3.}

- An invariant set $M$ in $\mathbb{R}^{3}$ is braidable if there exists an ambient isotopy which sends it into the standard solid torus $\mathcal{T}$ so that the flow is transverse to the fibers of $\mathcal{T}$.

- A set invariant $M$ in $\mathbb{S}^{3}$ is braidable if there exists a stereographic projection $S_{P}: \mathbb{S}^{3} \backslash\{P\} \rightarrow \mathbb{R}^{3}$, where $P$ is a point in the complement of $M$, such that $S_{P}(M)$ is braidable in $\mathbb{R}^{3}$.

In section 4 we will find two natural obstructions that prevent the braiding of nonsingular minimal sets in $\mathbb{R}^{3}$. One of them will enable us to construct a nonbraidable minimal set in $\mathbb{R}^{3}$. In the case when the dynamical system lies in $\mathbb{S}^{3}$, this one disappears (see subsection 4.2).

However, both obstructions vanishe when looking at any solenoid in $\mathbb{R}^{3}$ or in $\mathbb{S}^{3}$. Hence, as a particular case of our study, we get:

Corollary 1.4. Solenoids are braidable.

The two first sections will deal with geometric considerations about minimal sets and with a generalization of a classical theorem of knot theory. In appendix $\mathrm{A}$ we will prove propositions whose proofs involve concepts of knot theory.

Let us state precisely the fundamental properties of minimal sets used in this paper:

- the orbit of every point of a minimal set is dense in it (this is equivalent to Definition [1.1) ;

- a minimal (compact) set that is not a singularity admits a neighborhood that contains no singularity.

\section{Geometric propositions}

In this sequel $\left\{\phi_{t}\right\}$ is the flow of a smooth vector field $X$ (in $\mathbb{R}^{3}$ or in $\mathbb{S}^{3}$ ) and $M$ denotes a compact minimal set that is not a singularity.

A quite natural and necessary condition for $M$ being braidable is the existence of a disk having some particular properties that are given next.

Definition 2.1. A closed disk satisfies the section condition relative to $M$ if the restriction of the flow on $M$ crosses it every time on the same direction and if it intersects $M$ on its interior and not on its boundary.

So we will suppose that there exists a disk $\Delta$ that satisfies the section condition relative to $M$.

First, we have to describe the geometry of the compact set $K=M \cap \Delta$ to prove that the following configuration does not appear (see Figure11): the trajectory of a connected component of $K$ is knotted inside the cylinder defined by a trajectory of another connected component of $K$ that divides $\Delta$ in two connected components. In this situation, it is easy to see that $M$ is not braidable.

That is the purpose of Proposition 2.2

We recall some basic topological definitions. A set $\tilde{k}$ is a cut of $\Delta$ if its complement $\Delta \backslash \tilde{k}$ consists of at least two disjoint connected components. A subset of $\Delta$ is said to be nonbounded if one of its boundary components is in $\partial \Delta$.

Proposition 2.2. Suppose that $K$ has a connected component $\tilde{k}$ which is a cut of $\Delta$ and denote by $O$ one of the bounded connected components of $\Delta \backslash \tilde{k}$. Then, $O$ does not intersect $K$. 


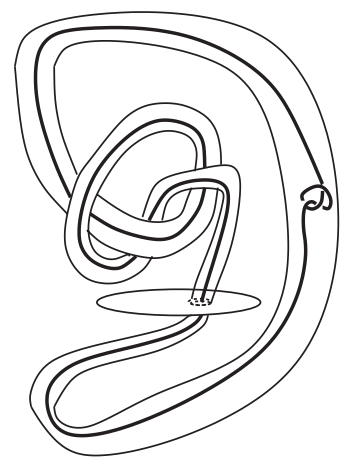

FiguRE 1. A forbidden configuration

Proof. Let $x$ be in $K$. Since $M$ is a nonsingular minimal set, the section condition implies that the orbit of $x$ intersects the interior of $\Delta$, int $(\Delta)$, at a finite time. By the continuity of $X$ (and the fact that $M$ is nonsingular), there exits a neighborhood of $x, V_{x}$, such that every point in $V_{x}$ returns to $\operatorname{int}(\Delta)$.

Because of compactness, we can extract a finite number of these open sets contained in $\operatorname{int}(\Delta)$ so that they form a finite cover of $K$.

Up to reparametrization, we can suppose that the first return time is equal to 1 for every point of this covering.

We will say that $\partial O$ is periodic if, up to the former time rescaling, there exists $N>0$ such that $\phi_{N}(\partial O)=\partial O$.

Lemma 2.3. If $O \cap K$ is not empty, then $\partial O$ is periodic.

Proof. Let $C$ be a connected component of $K$ contained in $O$. Choose a point $x_{0}$ in $C$ and an open neighborhood $V$ of $C$ whose closure is contained in $O$.

Since the orbit of $x_{0}$ is dense in $K$, it returns to $V$. Denote by $t_{0}$ the first time it happens. There exists an integer $N_{0}$ such that the arc of orbit of $x_{0}$ during time $t_{0}$ is contained inside the cylinder $\left\{\phi_{t}(\partial O), t \in\left[0, N_{0}\right]\right\}$.

Notice that $\phi_{N_{0}}(\partial O)$ is the boundary of one connected component of $\Delta \backslash K$ which is bounded, $U$.

There are then only three possibilities:

1. $V \subset O \cup \partial O \subset U$ (see the left side of Figure 2):

Since the orbit of $x_{0}$ accumulates $\partial U$, it intersects $U \backslash(O \cup \partial O)$. Choose a point $y_{0}$ of this intersection.

Since

$$
d\left(V ;\left\{\phi_{t}\left(y_{0}\right), t>0\right\} \cap \Delta\right)>d(V ; \partial O)>0,
$$

the orbit of $y_{O}$ cannot meet $V$. It refutes minimality.

2. $V \subset U \cup \partial U \subset O$ (see the right side of Figure 2)

Since

$$
d\left(\partial O ;\left\{\phi_{t}\left(x_{0}\right), t>0\right\} \cap \Delta\right)>\min _{i=1, \cdots, N_{0}} d\left(\partial O ; \phi_{i}(\partial U)\right)>d(\partial O ; \partial U)>0,
$$

the orbit of $x_{O}$ cannot accumulate $\partial O$, which is also impossible.

3. $\partial O$ is periodic.

Finally, the only possibility is the third one and so we proved the lemma. 


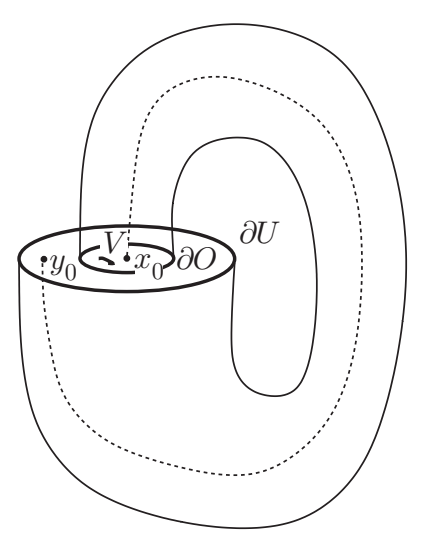

Figure 2. $V \subset O \cup \partial O \subset U$

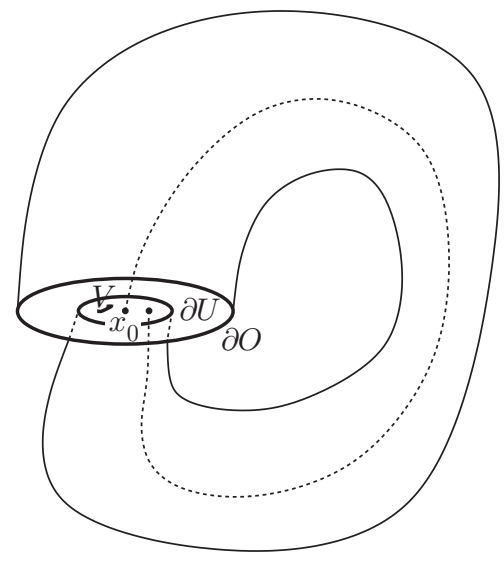

$V \subset U \cup \partial U \subset O$

Observe that if $\partial O$ is periodic, then minimality implies that $O$ cannot contain a point belonging to $K$. The end of the proof of the proposition is straightforward.

In fact, we can assert that

Proposition 2.4. There exists a covering of $K$ by connected open sets $\left\{O_{i}, i=\right.$ $1, \cdots, n\}$ satisfying the following conditions:

- their closures are pairwise disjoint and contained in int $(\Delta)$,

- their union returns to int $(\Delta)$,

- if $O_{i}$ is a cut of $\Delta$, then the bounded components of its complement do not contain any $O_{j}$.

Proof. Recall that a union of sets $\left\{S_{p}, p \in P\right\}$ forms a chain if for each $p$ in $P$ there exists $q$ in $P \backslash\{p\}$ such that $S_{q}$ is not disjoint from $S_{p}$.

Denote by $\left\{C_{i}, i=1, \cdots, m\right\}$ the covering of $K$ resulting from forming maximal chains with the open sets of the covering of $K$ appearing in Proposition 2.2. Notice that necessarily $K$ does not intersect the frontier of the open sets of this covering. Since $K$ is compact, for any $i$ in $\{1, \cdots, m\}$ the distance $\epsilon_{i}$ between $K$ and the frontier of the set $C_{i}$ is nonzero. So, removing from each $C_{i}$ an $\frac{\epsilon_{i}}{2}$-neighborhood of its frontier, we construct a covering of $K$ by a collection of open sets $\left\{O_{i}, i=\right.$ $1, \cdots, m\}$ satisfying the first two conditions of Proposition 2.4

Observe that if a set $S$ is not a cut of $\Delta$, then any two points of $\Delta \backslash S$ can be joined by a smooth arc in $\Delta$. Hence, if $O_{i}$ is a cut of $\Delta$ such that the bounded component of its complement contains an open set of the family, $O_{j}$, then either

- $O_{j} \cap K$ is not empty and therefore $O_{i}$ does not contain a component of $K$ which is a cut of $\Delta$ : we can replace $O_{i}$ by another open set which is not a cut of $\Delta$; or

- $O_{j} \cap K$ is empty and so we can remove $O_{j}$ from the covering.

So, Proposition 2.4 is a consequence of Proposition 2.2.

Notice that we can manage so that if $O_{i}$ is a cut of $\Delta$, then it contains a connected component of $K$ that is a cut of $\Delta$ also. 
The tubes resulting from the action of the flow on the covering of Proposition 2.4 look like a thick pinched knot which can be braided, and is explained in the next section.

\section{Pseudo-KNOTS}

Let $D$ be diffeomorphic to a euclidian embedded disk in the three-dimensional space $\mathbb{R}^{3}$ and let $\mathcal{G}=\left\{\gamma_{i}:[0,1] \rightarrow \mathbb{R}^{3}, i=1, \cdots, n\right\}$ be a finite collection of simple pairwise disjoint smooth curves satisfying the two following conditions (see Figure 3):

- each $\gamma_{i}$ intersects $D$ only on its endpoints and these intersections are transverse,

- each $\gamma_{i}^{\prime}(0)$ and each $\gamma_{i}^{\prime}(1)$ induces the same orientation on $D$.

We will denote by $|G|$ the union of the $\gamma_{i}([0,1])$.

Definition 3.1. The isotopy class under diffeomorphisms connected to Identity of such a knotted system will be called a pseudo-knot.

Definition 3.2. A pseudo-knot can be braided if there exist a representative (in its isotopy class) $(D, \mathcal{G})$ (which will be said to be braided) and an axis $A$ such that:

- $D$ is a euclidian disk,

- $A$ is contained in $\mathbb{R}^{3} \backslash(D \cup|G|)$,

- $A$ belongs to the 2-plane defined by $D$,

- each arc of $\mathcal{G}$ is parametrized by cylindrical coordinates relative to $A$, the polar angle functions being all increasing or all decreasing.

In classical knot theory, Alexander's theorem [Al] states that every knot is a closed braid. This fact remains true for pseudo-knots (see Figure 4).

Proposition 3.3. A pseudo-knot can be braided.

Proof. See Appendix, subsection A.1

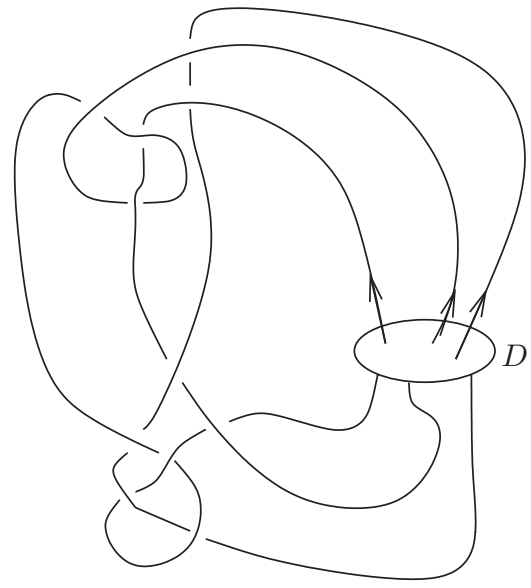

Figure 3. A representative of a pseudo-knot 


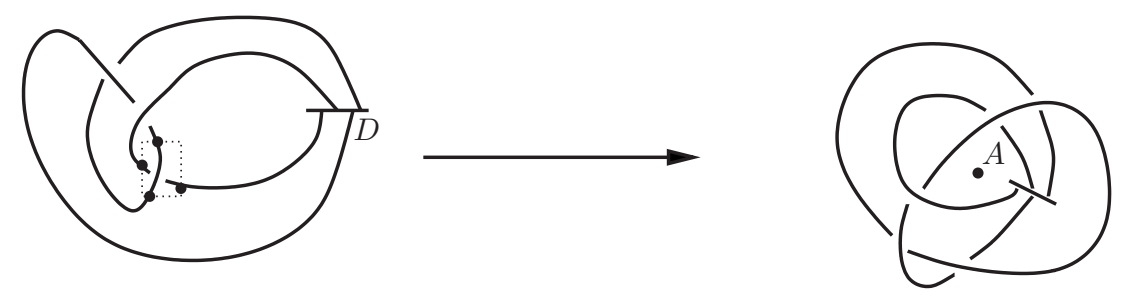

FiguRE 4. Braiding a pseudo-knot

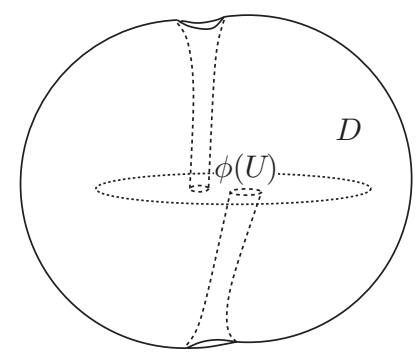

Figure 5. A fountain

Let $U$ be a connected open set of $\mathbb{R}^{2}$ and consider the tube $\bar{U} \times[0,1]$, where - stands for closure. A smooth map $\phi: \bar{U} \times[0,1] \rightarrow \mathbb{R}^{3}$ is a pseudo-embedding (relative to the disk $D$ ) if:

- $\left.\phi_{\mid \bar{U} \times] 0,1[}: \bar{U} \times\right] 0,1\left[\rightarrow \mathbb{R}^{3} \backslash D\right.$ is an embedding,

- $\phi(\bar{U} \times\{0\})$ and $\phi(\bar{U} \times\{1\})$ are contained in $D$.

There is a particular geometrical configuration (see Figure 5) that we have to take apart.

Definition 3.4. A pseudo-embedded tube $T=\phi(U \times[0,1])$ is a fountain (relative to $D$ ) if the frontier of $D$ lies in a bounded component of $\mathbb{R}^{3} \backslash T$.

Definition 3.5. A tubular pseudo-knot is a finite ordered collection of pseudoembedded tubes $\left\{\phi_{i}\left(\overline{U_{i}} \times[0,1]\right), i=1, \cdots, n\right\}$ such that:

- none of them is a fountain,

- they do not intersect each other except eventually on their opposite extremities,

- if $\phi_{i}\left(U_{i} \times\{0\}\right)$ is a cut of $D$, then the bounded components of its complement do not contain any $\phi_{j}\left(U_{j} \times\{0\}\right)$,

- if $x_{i}$ is in $U_{i}$ for $i=1 \cdots n$, then $\left\{\phi_{i}\left(\left\{x_{i}\right\} \times[0,1]\right), i=1, \cdots, n\right\}$ is a representative of a pseudo-knot.

It is easy to generalize Proposition 3.3 to tubular pseudo-knots by wrapping solid tubes (instead of strands) around a braid axis:

Corollary 3.6. A tubular pseudo-knot can be braided.

\section{BRAIDING Minimal SETS}

4.1. Braiding minimal sets of $\mathbb{R}^{3}$. Let $X$ be a smooth vector field in $\mathbb{R}^{3}$ and $M$ a nonsingular minimal set. Suppose that we get a disk $\Delta$ that satisfies the section 
condition. Up to the rescaling of Proposition 2.2, we can suppose that each point of $M \cap \Delta$ returns to $\Delta$ in time 1 .

We say that

Definition 4.1. $M$ forms a $\Delta$-fountain if the boundary of $\Delta$ is contained in a bounded component of $\mathbb{R}^{3} \backslash\left\{\phi_{t}(M \cap \Delta), t \in[0,1]\right\}$.

Now, notice that if $x, y$ are in $M \cap \Delta$, then the orientation induced by $X(x), X(y)$, $X\left(\phi_{1}(x)\right)$ and $X\left(\phi_{1}(y)\right)$ on $\Delta$ are the same. Hence, combined with Proposition 2.4 Corollary 3.6 implies the following theorem:

Theorem 4.2. Let $X$ be a smooth vector field of the 3-dimensional euclidian space and consider $M$ a minimal set that is not a singularity.

If there exists a disk $\Delta$ satisfying the section condition relative to $M$ such that $M$ is not a $\Delta$-fountain, then $M$ is braidable.

It is important to notice that the existence of a disk satisfying the section condition is not sufficient to braid a minimal set:

Proposition 4.3. There exists a nonbraidable minimal set of a smooth flow on $\mathbb{R}^{3}$ for which we can find a disk satisfying the section condition.

Proof. See Appendix, subsection A.2

However, notice that the irrational flow on the trivial torus $\mathbb{T}^{2}=\partial \mathcal{T}$ is braidable even though there exists a disk that satisfies the section condition and for which the trivial torus is a fountain: choose a disk whose boundary is the core of the torus, then the intersection of $\mathbb{T}^{2}$ with this disk forms a fountain.

Observe that the proof of Theorem 4.2 given here allows us to braid not only a nonsingular minimal set but a tubular neighborhood of it.

4.2. Braiding minimal sets of $\mathbb{S}^{3}$. In this subsection, we consider a minimal set $M$ of a flow on $\mathbb{S}^{3}$. We suppose that it is different from a singularity and from the 3 -sphere. In the last case, it is obvious that the minimal set is not braidable. However, it is not known if such a flow exists.

The latter condition allows us, without loss of generality, to suppose that the dynamical system lies in $\mathbb{R}^{3}$. Choose a point $P_{\infty}$ (we will call it the infinity point) in the complement of $M$ and of the singularities of the flow and then consider the (smooth) stereographic projection $S_{P_{\infty}}: \mathbb{S}^{3} \backslash P_{\infty} \rightarrow \mathbb{R}^{3}$; it sends our dynamical system in $\mathbb{R}^{3}$ so that $S_{P_{\infty}}(M)$ remains a nonsingular minimal set of the image of the flow. So we can apply Theorem 4.2 to it.

Notice that every connected component of the complement of any set of $\mathbb{S}^{3}$ is bounded and thus the fountain condition makes no sense in $\mathbb{S}^{3}$.

In fact, we will prove the following theorem:

Theorem 4.4. Let $X$ be a smooth vector field of the 3-dimensional sphere and consider $M$ a minimal set that is not a singularity.

If there exists a disk satisfying the section condition relative to $M$, then $M$ is braidable.

Proof. Let $\Delta$ be a disk satisfying the section condition relative to $M$.

Consider the covering $\left\{O_{i}, i=1, \cdots, n\right\}$ of $K=M \cap \Delta$ resulting from Proposition 2.4 Denote by $T$ the union of the tubes (up to reparametrization) $T_{i}=$ $\left\{\phi_{t}\left(O_{i}\right), t \in[0,1]\right\}$. The statement of Proposition 2.4 implies that the boundary of 
$\Delta$ is contained in one of the connected components of the complement of $T$. Choose the infinite point in this connected component.

Consider the corresponding stereographic projection. It sends our systems in $\mathbb{R}^{3}$ so that the resulting dynamical system satisfies the hypothesis of Theorem 4.2 .

It is worthwhile to note that the statement of Theorem 4.4 is already known in the case when the minimal set is hyperbolic. In this case the dynamics reduces to the dynamics on a template, i.e. a branched 2-manifold, and Franks and Williams (see FrWi]) proved that such templates can be braided. However, Theorem 4.4 remains valid in a more general context: it seems that the existence of a disk satisfying the section condition is not a strong one.

\section{Appendix A. Proofs involving Knot theory}

A.1. Proof of Proposition 3.3. We will prove that a pseudo-knot can be braided.

The proof is just an extension of the one for classical knots. Therefore, more details can be found in [Al].

Let $(D, \mathcal{G})$ be a representative of a given pseudo-knot. Choose an axis $A$ in $\mathbb{R}^{3} \backslash(D \cup|G|)$.

We can manage so that:

- $D$ is a euclidian disk,

- $|G|$ is defined by a diagram in the $(r, \theta)$-plane relative to $A$,

- there exists a neighborhood $N$ of $D$ such that $\theta_{\mid N \cap \gamma_{i}}$ are all increasing or all decreasing (suppose increasing).

Let $\Lambda$ be a finite collection of smooth curves satisfying the following three conditions:

- their union equals to $|G|$,

- two of them can intersect only on their endpoints,

- each of them contains at most a crossing point.

Moreover, we can assume modulo an isotopy that $\theta_{\mid \lambda}$ is nonconstant for each $\lambda$ in $\Lambda$. There are then two types of arcs: positive ones if $\theta_{\mid \lambda}$ is increasing and negative ones if $\theta_{\mid \lambda}$ is decreasing.

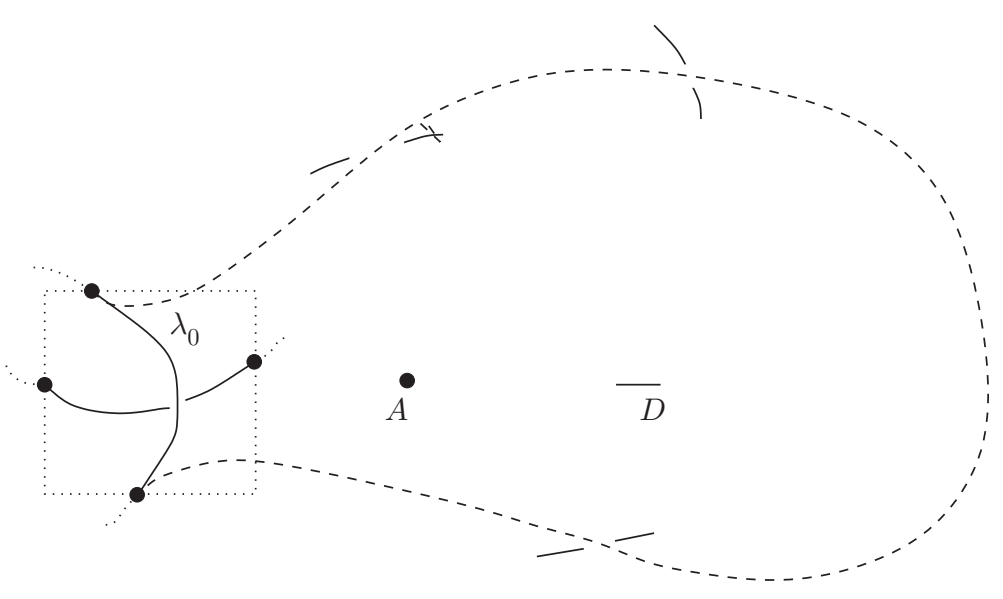

FiguRE 6 . Replacement of a bad arc by good arcs 


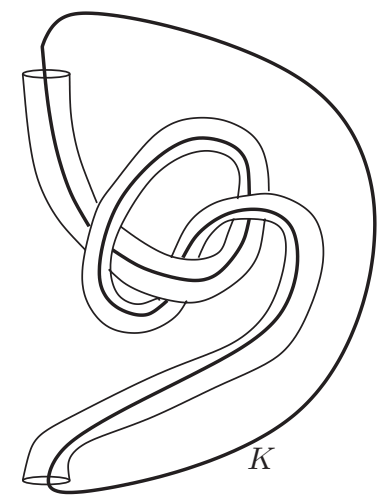

Figure 7. A knotted tube

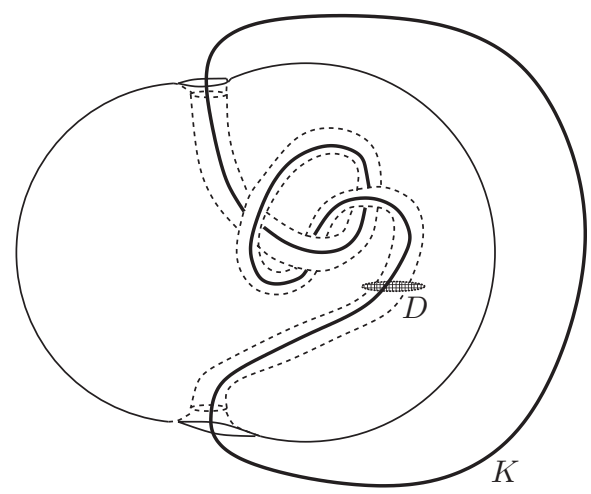

The fountain after gluing the tube

If $\lambda_{0}$ is a negative arc, then replace it (via an isotopy) by a positive one, which respect the type of crossing of $\lambda_{0}$ (see Figure 6). Then the number of negative arcs decreases.

This finite process yields to a braided representative.

Notice that without the second condition defining knotted systems, the proof does not work. In fact, without this assumption, Proposition 3.3 is not true.

A.2. Proof of Proposition 4.3. We want to construct a minimal set in $\mathbb{R}^{3}$ that cannot be braided and for which there exists a disk $D$ that satisfies the section condition.

Let $K$ be a knot in $\mathbb{R}^{3}$. Construct a knotted tube as follows (see the left side of Figure 7): choose a planar part of $K$ and consider a tubular neighborhood of its complement. Now glue together the extremities of this tube so that it forms a fountain $\mathcal{F}_{K}$ (relative to a disk $D$ which is transverse to our previous tube); see the right side of Figure 7 Such a fountain will be called a K-type fountain.

Lemma A.1. A $K$-type fountain $\mathcal{F}_{K}$ is braidable if and only if $K$ is unknotted.

Proof. If we denote by $\mathcal{V}_{K}$ the bounded complement of this fountain in $\mathbb{R}^{3}$, we can prove that $\mathcal{F}_{K}$ is not braidable by showing that the fundamental group of $\mathbb{R}^{3} \backslash \mathcal{V}_{K}$ is not equal to the one of $\mathbb{D}^{2} \times \mathbb{S}^{1}$, i.e., $\pi_{1}\left(\mathbb{R}^{3} \backslash \mathcal{V}_{K}\right) \neq \mathbb{Z}$.

Consider $\mathbb{S}^{3}$ as the natural compactification of $\mathbb{R}^{3}$. Remark that $\mathcal{V}_{K}$ is now a tubular neighborhood of $K$. So the knot group of $K$ (the fundamental group of the complement of the knot) is equal to $\pi_{1}\left(\mathbb{R}^{3} \backslash \mathcal{V}_{K}\right)$.

Recall that Dehn's lemma implies the following theorem (see [Ro]), known as the unknotting theorem:

Theorem A.2. A knot is trivial if and only if its knot group is $\mathbb{Z}$.

This achieves the proof of lemma.

Given a $K$-type fountain $\mathcal{F}_{K}$ we always can construct a smooth flow $\left\{\phi_{t}\right\}$ on $\mathbb{R}^{3}$ such that the restriction of $\left\{\phi_{t}\right\}$ on $\mathcal{F}_{K}$ is an irrational flow. So, to each nontrivial knot, we can assign a vector field in $\mathbb{R}^{3}$ that satisfies the properties of Proposition 4.3 . 


\section{ACKNOWLEDGMENTS}

It is a pleasure to thank the careful unknown referee of the first version of this paper.

\section{REFERENCES}

[Al] Alexander, J.W.: A lemma on systems of knotted curves, Proc. Nat. Acad. Sci. U.S.A. 9, (1923), 93-95.

[ArKh] Arnold, V.I. \& Khesin, B.: Topological methods in hydrodynamics, Applied Mathematical Sciences 125, (1998). MR 99b:58002

[Bi] Birkhoff, G. D.: Dynamical systems, AMS, Providence, RI, (1927), reprint (1966). MR 35:1

[BiWi1] Birman, J.S. \& Williams, R.F.: Knotted periodic orbits in dynamical systems. I. Lorenz's equations, Topology 22, (1983). MR 84k:58138

[BiWi2] Birman, J.S. \& Williams, R.F.: Knotted periodic orbits in dynamical system. II. Knot holders for fibered knots, Contemp. Math. 20, (1983). MR 86a:58084

[Bo] Boyland, P.: Topological methods in surface dynamics, Topology Appl. 58, (1994). MR 95h:57016

[CMAn] Casasayas, J. \& Martinez Alfaro, J. \& Nunes, A.: Knot and links in integrable hamiltonian systems, J. Knot Theory Ramifications 7, (1998). MR 99f:58111

[FrWi] Franks, J. \& Williams, R.F.: Entropy and knots, Trans. Amer. Math. Soc. 291, (1985). MR 86k:58105

[GaG1] Gambaudo, J.-M. \& Ghys É.: Enlacements asymptotiques, Topology 36, (1997). MR 98f: 57050

[GaG2] Gambaudo, J.-M. \& Ghys É.: Signature asymptotique d'un champ de vecteurs en dimension 3, Duke Math. J. 106, (2001). CMP 2001:07

[GST1] Gambaudo, J.-M. \& van Strien, S. \& Tresser, C.: The periodic orbit structure of orientation preserving diffeomorphisms on $D^{2}$ with topological entropy zero, Ann. Inst. Henri Poincaré 49, (1989). MR 90j:57008

[GST2] Gambaudo, J.-M. \& van Strien, S. \& Tresser, C.: Vers un ordre de Sarkovskiŭ pour les plongements du disque préservant l'orientation, C. R. Acad. Sci. Paris Sér. I Math. 310, (1990) MR 91f:58072

[GhHo] Ghrist, R.W. \& Holmes, P.J.: An ODE whose solutions contain all knots and links, Intl. J. Bifurcation and Chaos 5, (1996). MR 97j:58127

[Gh] Ghys, E.: Construction de champs de vecteurs sans orbite périodique (d'après Krystyna Kuperberg), Astérisque 227, (1995). MR 95k:57032

[KaHa] Katok, A. \& Hasselblatt, B.: Introduction to the modern theory of dynamical systems, Encyclopedia of mathematics and its applications 54, (1995). MR 96c:58055

[Ko] Kolev, B.: Dynamique topologique en dimension 2. Orbites périodiques et entropie topologique, Ph.D. Thesis, (1991).

[Ku] Kuperberg, K.: A smooth counterexample to the Seifert conjecture, Ann. of Math. 140, (1994). MR 95g:57040

[Mu Murasugi, K. Knot theory and its applications. Translated from the 1993 Japanese original by Bohdan Kurpita. Birkhäuser Boston, Inc., Boston, (1996). MR 97g:57011

[Po] Poincaré, H.: Nouvelles méthodes de la mécanique céleste, Gauthier-Villars, Paris, (1899).

[Ro] Rolfsen, D.: Knots and links. Corrected reprint of the 1976 original, Mathematics Lecture Series 7, (1990). MR 95c:57018

[Sm] Smale, S.: Differential dynamical systems, Bull. Am. Math. Soc. 73, (1967).

[Wa] WADA, M.: Closed orbits of nonsingular Morse-Smale flows on $\mathbb{S}^{3}$, J. Math. Soc. Jap. 41, (1989). MR 90g:58059

Laboratoire de Topologie, Université de Bourgogne, UMR CNRS 5584, B.P. 47870 21078, Dijon Cedex, France

E-mail address: lagrange@topolog.u-bourgogne.fr 